\title{
PLACENTA IN PREGNANCY-INDUCED HYPERTENSION- HISTOPATHOLOGY AND FOETAL OUTCOME- A CORRELATIVE STUDY
}

\author{
Kavitha Ravi ${ }^{1}$, Santha Sadasivan ${ }^{2}$
}

${ }_{1}^{1}$ Associate Professor, Department of Pathology, Government Medical College, Ernakulam, Kerala, India.

${ }^{2}$ Professor and HOD (Retired), Department of Pathology, Government Medical College, Thiruvananthapuram, Kerala, India.

\begin{abstract}
BACKGROUND
The Aims and Objectives of our study were-

1. To study the gross and histological changes in the placenta in pregnancy-induced hypertension.

2. To correlate the placental changes with the severity of pregnancy-induced hypertension.

3. To correlate the placental changes with the foetal outcome.
\end{abstract}

ABSTRACT

Placenta is an organ specially formed for the purpose of supplying oxygen and nutrition to the foetus. In addition, it accomplishes the functions of excretion and haematopoiesis and secretes a number of hormones all directly related to foetal nutrition and aimed at preparing the foetus for extrauterine life. ${ }^{1}$ Pregnancy-induced hypertension (PIH) is one among the most common complications in pregnancy. Severe pregnancy-induced hypertension is a serious health hazard to both the mother and her foetus. Various studies undertaken on placenta in PIH have revealed increased incidence of certain typical, but not pathognomonic lesions. ${ }^{2}$ The most consistent findings were cytotrophoblastic proliferation, thickening of the villous basement membrane and paucity of vasculosyncytial membrane. ${ }^{3}$ These are suggestive of a reduced uteroplacental blood flow, which may be predicted by Doppler studies. ${ }^{4}$ Also the technique of placental bed biopsy enables a more precise assessment of the uteroplacental vasculature. ${ }^{5}$ Regarding the foetal outcome, the incidence of intrauterine growth restriction and perinatal mortality has been found to be more in PIH compared to normal pregnancy. The hypertension alone may not affect the foetal outcome, but its coexistence with the placental lesions result in growth restriction and/or foetal loss. ${ }^{6}$ The present study was undertaken to assess the pattern of histopathological changes in the placenta and the foetal outcome in relation to the severity of pregnancy-induced hypertension.

\section{MATERIALS AND METHODS}

Our study was a cross-sectional study conducted at the Department of Pathology, Medical College, Thiruvananthapuram and Department of Obstetrics and Gynaecology, SAT Hospital, Thiruvananthapuram for a period of 2 years. Fifty placentas from patients with pregnancy-induced hypertension and an equal number from normal pregnancy were studied. Cases with multiple pregnancy, anaemia, past history of hypertension, history of renal disease, history of chronic vascular disease and diabetes mellitus were excluded from the study. The relevant clinical details regarding the mother and the baby were obtained as per the structured proforma. The placentas were collected soon after delivery, fixed whole in $10 \%$ formalin for two weeks and then examined grossly and microscopically. The paraffin sections were stained with Haematoxylin and Eosin, PAS and Van Gieson stain.

The villous lesions noted were-

1. Cytotrophoblastic proliferation.

2. Basement membrane thickening.

3. Paucity of vasculosyncytial membrane.

4. Fibrinoid necrosis.

5. Excessive syncytial knots.

6. Stromal fibrosis.

\section{RESULTS}

In the present study, 50 placentas from patients with pregnancy-induced hypertension and an equal number from normal pregnancy were studied. The placental histopathology was correlated with the foetal outcome. Depending on the blood pressure, presence of oedema, albuminuria and convulsions the study group was divided into-

1. Mild PIH, 25 cases.

2. Severe PIH, 18 cases.

3. Eclampsia, 7 cases.

The peak incidence of pregnancy-induced hypertension was in the age group of 20 - 29 years (82\%). The incidence of PIH was more in primigravidae (58\%). Only 6\% in the study group were G3 or above. The mean placental weight in the control group was 464 grams, while it was 415 grams in the study group. In the normal group and mild PIH, the mean weight was comparable whereas it was significantly lower in cases of severe PIH and eclampsia. Considering the grade of PIH the mild group showed no appreciable difference from the control group, whereas the mean infarction (Percentage) in the severe and eclampsia group was significantly higher. The incidence of retroplacental clot was higher in the study group compared to the control group, which was statistically highly significant. There was no significant difference in the incidence of calcification and intervillous thrombus between the study group and the control group. The incidence of cytotrophoblastic proliferation and basement membrane thickening was much higher in the study group than in the control group. The other lesions which showed an increased incidence in the study group were paucity of vasculosyncytial membrane, fibrinoid necrosis of the villi, excessive syncytial knots and villous stromal fibrosis. In the present study the incidence of the following placental lesions was found to be directly related to the severity of PIH, infarction, retroplacental clot, cytotrophoblast proliferation, basement membrane thickening, paucity of vasculosyncytial membrane, excessive syncytial knots and stromal fibrosis. The incidence of preterm delivery in the study group was more than 2 times higher than that in the control group. Thus, the observations in the present study indicate that the placental 
histopathology is directly related to the severity of PIH and the adverse foetal outcome is well correlated with the extent of infarction and the villous lesions in the placenta.

\section{CONCLUSION}

The placental histopathological findings observed in this study suggest the existence of a reduced uteroplacental blood flow in pregnancy-induced hypertension. The abnormal placentation in PIH has been well established by many workers by their studies on the placental bed vasculature. As a response to the uteroplacental ischaemia there is vasoconstriction of the foetal stem arteries in the placenta, manifesting histologically as sclerosis of vessels and increased number of syncytial knots. The intrauterine growth retardation and foetal loss observed in PIH are also attributable to the defective uteroplacental perfusion. The fact that the placental bed vessels, which harbour the important pathological processes are not usually available for study is particularly distressing. However, placental histopathology with more emphasis on the technique of placental bed biopsy could compliment the clinical diagnosis or support the clinical suspicion in cases, in which the blood pressure values do not meet the criteria for a diagnosis of preeclampsia.

\section{KEY WORDS}

Placental Pathology, PIH, Foetal Outcome.

HOW TO CITE THIS ARTICLE: Ravi K, Sadasivan S. Placenta in pregnancy-induced hypertension- histopathology and foetal outcome- a correlative study. J. Evolution Med. Dent. Sci. 2018;7(23):2761-2766, DOI: 10.14260/jemds/2018/624

\section{BACKGROUND}

The placenta is an organ specially formed for the purpose of supplying oxygen and nutrition to the foetus; in addition it accomplishes the functions of excretion and haematopoiesis and secretes a number of hormones, all directly related to foetal nutrition and aimed at preparing the foetus for extrauterine life. 1 As Benirschke, an expert in placental pathology has stated- The placenta is the most accurate record of the infant's prenatal experiences. ${ }^{2}$ The long-term goal of examining placentas from patients with adverse pregnancy outcomes have always been to understand better the basic underlying disease processes involved.

Pregnancy-induced hypertension (PIH) is one among the most common complications in pregnancy. The diagnosis of $\mathrm{PIH}$ is made when the blood pressure is $140 / 90 \mathrm{mmHg}$ or greater during the second half of pregnancy in a previously normotensive woman, severe pregnancy induced. Hypertension is a serious health hazard to both the mother and her foetus. ${ }^{3}$

The observations that PIH can complicate pregnancies without a foetus (As in vesicular mole) and can occur in abdominal pregnancies (i.e. independent of uterus have led to this condition being termed as a placental disorder). The fact that delivery with the removal of the placenta has been the ultimate cure of the condition that further supports this conclusion. Various studies undertaken on placenta in PIH have revealed increased incidence of certain typical, but not pathognomonic lesions. The most consistent findings were cytotrophoblastic proliferation, thickening of the villous basement membrane and paucity of vasculosyncytial membrane. These are suggestive of a reduced uteroplacental blood flow, which may be predicted by Doppler studies. ${ }^{4}$ Also the technique of placental bed biopsy enables a more precise assessment of the uteroplacental vasculature. 5

'Financial or Other Competing Interest': None.

Submission 20-03-2018, Peer Review 21-05-2018,

Acceptance 28-05-2018, Published 04-06-2018.

Corresponding Author:

Dr. Kavitha Ravi,

Karthika, KMLRA 24,

Mosque Lane, Kumarapuram,

Thiruvananthapuram, Kerala, India.

E-mail: kavitharavibinoy@gmail.com

DOI: $10.14260 /$ jemds $/ 2018 / 624$
Regarding the foetal outcome, the incidence of intrauterine growth restriction and perinatal mortality has been found to be more in PIH compared to normal pregnancy. The hypertension alone may not affect the foetal outcome, but its coexistence with the placental lesions result in growth restriction and/or foetal loss. ${ }^{6}$ The present study was undertaken to assess the pattern of histopathological changes in the placenta and the foetal outcome in relation to the severity of pregnancy-induced hypertension.

\section{Objectives of Study}

1. To study the gross and histological changes in the placenta in pregnancy-induced hypertension.

2. To correlate the placental changes with the severity of pregnancy-induced hypertension.

3. To correlate the placental changes with the foetal outcome.

\section{MATERIALS AND METHODS \\ Study Design}

Cross-sectional study.

\section{Setting}

Department of Pathology, Medical College, Thiruvananthapuram and Department of Obstetrics and Gynaecology, SAT Hospital, Thiruvananthapuram for a period of 2 years.

\section{Materials}

Fifty placentas from patients with pregnancy-induced hypertension and an equal number from normal pregnancy were studied.

The study group was again subdivided into mild PIH, severe PIH and eclampsia on the basis of the level of blood pressure after completion of 28 weeks of gestation, presence of pedal oedema, albuminuria and convulsions. Cases with multiple pregnancies, anaemia, past history of hypertension, history of renal disease, history of chronic vascular disease and diabetes mellitus were excluded from the study.

\section{Methodology}

The relevant clinical details regarding the mother and the baby were obtained per the structured proforma 
(Appendix I). The placentas were collected soon after delivery, fixed whole in $10 \%$ formalin for two weeks and then examined.

\section{Examination of Placenta}

On initial examination the shape of the placenta, the type of insertion of cord and abnormalities of the cord were noted. The weight of the placenta was recorded after cutting off the cord 2 to $4 \mathrm{~cm}$ from its insertion. The cut end of the cord was examined for the number of vessels. The extraplacental membranes, the foetal surface and the maternal surface were examined for any abnormality with special attention to the presence of retroplacental clots, calcification and infarction. ${ }^{2}$

The findings were recorded in the proforma. The entire placenta was then cut into vertical strips, each of $0.5 \mathrm{~cm}$ thickness and any gross pathology noted. Two bits each from the central and peripheral area were taken. Additional sections were taken from grossly abnormal areas. Transverse section of the cord and membrane roll were also taken for histological examination.

The paraffin sections were stained with Haematoxylin and Eosin, PAS and Van Gieson stain. PAS staining was done to study the basement membrane changes and Van Gieson stain to demonstrate stromal fibrosis.

\section{The Villous Lesions noted were-}

1. Cytotrophoblastic proliferation.

2. Basement membrane thickening.

3. Paucity of vasculosyncytial membrane.

4. Fibrinoid necrosis.

5. Excessive syncytial knots.

6. Stromal fibrosis.

For a quantitative result, 100 villi in the maternal zone of each of four sections were counted and the number of villi showing the villous lesion was expressed as a percentage of incidence.

\section{Statistical Analysis}

The data collected were coded and entered in Microsoft Excel and statistical analysis was done using SPSS Version 16. Quantitative variables were summarised as Mean and Standard deviation, while qualitative variables as frequency and percentage.

Chi-square test and ' $t$ ' test were used to find out statistical significance. Significance level was kept at $5 \%$.

\section{RESULTS}

In the present study, 50 placentas from patients with pregnancy-induced hypertension and an equal number from normal pregnancy were studied. The placental histopathology was correlated with the foetal outcome. Depending on the blood pressure, presence of oedema, albuminuria and convulsions, the study group was divided into Mild PIH 25 cases, Severe PIH 18 cases and Eclampsia 7 cases. The peak incidence of pregnancy-induced hypertension was in the age group of $20-29$ years $(82 \%)$ gravidity. The incidence of PIH was more in primigravidae (58\%). Only $6 \%$ in the study group were G3 or above.

\section{Placental Histopathology/ Weight}

The mean placental weight in the control group was 464 grams, while it was 415 grams in the study group. In the normal group and mild PIH the mean weight was comparable, whereas it was significantly lower in cases of severe PIH and eclampsia. ${ }^{7}$

\begin{tabular}{|c|c|c|c|c|c|}
\hline \multirow{2}{*}{ Group } & \multirow{2}{*}{$\mathbf{N}$} & \multicolumn{2}{|c|}{ Placental Weight Gms } & $\begin{array}{c}\mathbf{p} \\
\text { value }\end{array}$ & $\begin{array}{c}\mathbf{p} \\
\text { value }\end{array}$ \\
\cline { 3 - 6 } Control & 50 & 464 & 52 & - & - \\
\hline Study & 50 & 415 & 101 & 3.05 & $\mathrm{P}<.01$ \\
\hline (1) Mild & 25 & 490 & 70 & 1.83 & $\mathrm{P}>.05$ \\
\hline (2) Severe & 18 & 363 & 53 & 7.93 & $\mathrm{P}<.001$ \\
\hline (3) Eclampsia & 7 & 274 & 48 & 14.33 & $\begin{array}{c}\mathrm{P}< \\
.0001\end{array}$ \\
\hline \multicolumn{7}{|c|}{ Table 1. Placenta Weight and Level of Significance } \\
\hline
\end{tabular}

\section{Infarction}

The mean infarction percentage in the study group was 16.85 compared to 5.25 in the control group, which was found to be highly significant statistically $(\mathrm{t}=6.3$, d. $\mathrm{f}=98, \mathrm{p}=$ less than 0.001). Considering the grade of PIH the mild group showed no appreciable difference from the control group, whereas the mean infarction (percentage) in the severe and eclampsia group was significantly higher. ${ }^{8}$

\begin{tabular}{|c|c|c|c|c|}
\hline \multirow{2}{*}{ Group } & \multicolumn{2}{|c|}{ Infarction (\%) } & \multirow{2}{*}{ T value } & \multirow{2}{*}{ P value } \\
\cline { 2 - 5 } & Mean & S.D & & $\mathrm{P}<.001$ \\
\hline Control & 5.25 & 3.98 & 6.30 & \\
\hline Study & 16.85 & 12.40 & & \\
\hline (i) Mild & 6.10 & 3.60 & 0.97 & $\mathrm{P}>.05$ \\
\hline (ii) Severe & 16.70 & 7.9 & 7.53 & $\mathrm{P}<.001$ \\
\hline (iii) Eclampsia & 27.9 & 5.7 & 17.42 & $\mathrm{P}<.0001$ \\
\hline \multicolumn{6}{|c|}{ Table 2. Mean and SD of Infarction (\%) and Level of } \\
Significance \\
\hline
\end{tabular}

Distribution of other gross lesions. The incidence of retroplacental clot was higher in the study group compared to the control group, which was statistically highly significant. There was no significant difference in the incidence of calcification and intervillous thrombus between the study group and the control group.

\begin{tabular}{|c|c|c|c|c|c|c|}
\hline \multirow{2}{*}{ Lesion } & \multicolumn{2}{|c|}{$\begin{array}{c}\text { Control } \\
\text { Group }\end{array}$} & \multicolumn{2}{c|}{$\begin{array}{c}\text { Study } \\
\text { Group }\end{array}$} & \multirow{2}{*}{$\begin{array}{c}\text { Z } \\
\text { value }\end{array}$} & value \\
\cline { 2 - 6 } & No & $\mathbf{\%}$ & No & $\mathbf{\%}$ & & \\
\hline Retroplacental clot & 1 & 2 & 17 & 34 & 4.58 & $\mathrm{p}<.01$ \\
\hline Calcification & 38 & 76 & 32 & 64 & 0.01 & $\mathrm{p}>.05$ \\
\hline $\begin{array}{c}\text { Intervillous } \\
\text { thrombus }\end{array}$ & 11 & 22 & 19 & 38 & 1.77 & $\mathrm{p}>.05$ \\
\hline
\end{tabular}

Distribution of microscopic villous lesions, the incidence of cytotrophoblastic proliferation and basement membrane thickening was much higher in the study group than in the control group. Statistical tests proved this difference to be highly significant. The other lesions which showed an increased incidence in the study group were paucity of vasculosyncytial membrane, fibrinoid necrosis of the villi, excessive syncytial knots and villous stromal fibrosis. PAS stain highlighted the thickened basement membrane, cytotrophoblast and fibrinoid necrosis. Van Gieson stain demonstrated the villous stromal fibrosis. The villous lesions in the mild group were comparable to those in the control group. But the $\%$ of incidence of the lesions in the severe and 
eclampsia groups were much higher compared to the control group. Thus, in the present study the incidence of the following placental lesions was found to be directly related to the severity of PIH, infarction, retroplacental clot, cytotrophoblast proliferation, basement membrane thickening, paucity of vasculosyncytial membrane, excessive syncytial knots and stromal fibrosis.

\begin{tabular}{|c|c|c|c|c|c|c|c|}
\hline \multicolumn{2}{|c|}{ Villous Lesion (\%) } & \multicolumn{2}{|c|}{ Control } & \multicolumn{2}{|c|}{ Study } & \multirow[t]{2}{*}{$\mathrm{X}^{2}$} & \multirow[t]{2}{*}{ P value } \\
\hline & & No. & $\%$ & No. & $\%$ & & \\
\hline CT proliferation & $\begin{array}{c}<20 \\
20-40 \\
>40\end{array}$ & $\begin{array}{c}48 \\
2 \\
-\end{array}$ & $\begin{array}{c}96 \\
4 \\
-\end{array}$ & $\begin{array}{c}24 \\
18 \\
8\end{array}$ & $\begin{array}{l}48 \\
36 \\
16\end{array}$ & 26.24 & $\mathrm{p}<.001$ \\
\hline BM thickening & $\begin{array}{l}<3 \\
>3\end{array}$ & $\begin{array}{c}46 \\
4\end{array}$ & $\begin{array}{c}92 \\
8\end{array}$ & $\begin{array}{l}24 \\
26\end{array}$ & $\begin{array}{l}48 \\
52\end{array}$ & 21.0 & $\mathrm{p}<.001$ \\
\hline $\begin{array}{l}\text { Vasculosyncytial } \\
\text { membrane }\end{array}$ & $\begin{array}{l}<6 \\
6-30\end{array}$ & $\begin{array}{c}2 \\
48\end{array}$ & $\begin{array}{c}4 \\
96\end{array}$ & $\begin{array}{l}13 \\
37\end{array}$ & $\begin{array}{l}26 \\
74\end{array}$ & 7.84 & $\mathrm{p}<.01$ \\
\hline $\begin{array}{c}\text { Fibrinoid } \\
\text { necrosis }\end{array}$ & $\begin{array}{l}<3 \\
>3 \\
\end{array}$ & $\begin{array}{l}32 \\
18 \\
\end{array}$ & $\begin{array}{l}64 \\
36 \\
\end{array}$ & $\begin{array}{l}14 \\
36 \\
\end{array}$ & $\begin{array}{l}28 \\
72 \\
\end{array}$ & 11.6 & $\mathrm{p}<.01$ \\
\hline $\begin{array}{l}\text { Syncytial } \\
\text { knots }\end{array}$ & $\begin{array}{c}>30 \\
30-50 \\
>50\end{array}$ & $\begin{array}{c}32 \\
15 \\
3\end{array}$ & $\begin{array}{c}64 \\
30 \\
6\end{array}$ & $\begin{array}{l}12 \\
26 \\
12\end{array}$ & $\begin{array}{l}24 \\
52 \\
24\end{array}$ & 14.6 & $\mathrm{p}<.01$ \\
\hline $\begin{array}{l}\text { Stromal } \\
\text { fibrosis }\end{array}$ & $\begin{array}{l}<6 \\
>6\end{array}$ & $\begin{array}{c}49 \\
1\end{array}$ & $\begin{array}{c}98 \\
2\end{array}$ & $\begin{array}{l}38 \\
12\end{array}$ & $\begin{array}{l}76 \\
24\end{array}$ & 8.84 & $\mathrm{p}<.01$ \\
\hline
\end{tabular}

\begin{tabular}{|c|c|c|c|c|c|c|c|c|c|}
\hline \multicolumn{2}{|c|}{ Villous Lesion (\%) } & \multicolumn{2}{|c|}{ Mild (25) } & \multicolumn{2}{|c|}{ Severe (18) } & \multicolumn{2}{|c|}{ Eclampsia (7) } & \multirow[t]{2}{*}{$\mathrm{X}^{2}$} & \multirow[t]{2}{*}{ P value } \\
\hline & & No. & $\%$ & No. & $\%$ & No. & $\%$ & & \\
\hline CT proliferation & $\begin{array}{c}<20 \\
20-40 \\
>40\end{array}$ & $\begin{array}{c}21 \\
4 \\
-\end{array}$ & $\begin{array}{c}84 \\
16 \\
-\end{array}$ & $\begin{array}{c}3 \\
12 \\
3\end{array}$ & $\begin{array}{l}16 \\
67 \\
17\end{array}$ & $\begin{array}{l}- \\
2 \\
5\end{array}$ & $\begin{array}{c}- \\
29 \\
71\end{array}$ & 15.5 & $\mathrm{p}<.01$ \\
\hline BM thickening & $\begin{array}{l}<3 \\
>3\end{array}$ & $\begin{array}{c}21 \\
4\end{array}$ & $\begin{array}{l}84 \\
16\end{array}$ & $\begin{array}{c}3 \\
15\end{array}$ & $\begin{array}{l}17 \\
83\end{array}$ & $\overline{7}$ & $\begin{array}{c}- \\
100\end{array}$ & 51 & $\mathrm{p}<.001$ \\
\hline $\begin{array}{c}\text { Vasculosyncytial } \\
\text { membrane }\end{array}$ & $\begin{array}{c}<< \\
6-30\end{array}$ & $\begin{array}{c}1 \\
24 \\
\end{array}$ & $\begin{array}{c}4 \\
96 \\
\end{array}$ & $\begin{array}{c}6 \\
12 \\
\end{array}$ & $\begin{array}{l}33 \\
67 \\
\end{array}$ & $\begin{array}{l}6 \\
1 \\
\end{array}$ & $\begin{array}{l}85.7 \\
14.3 \\
\end{array}$ & 10.39 & $\mathrm{p}<.01$ \\
\hline $\begin{array}{c}\text { Fibrinoid } \\
\text { necrosis }\end{array}$ & $\begin{array}{l}<3 \\
>3\end{array}$ & $\begin{array}{l}14 \\
11\end{array}$ & $\begin{array}{l}56 \\
44 \\
\end{array}$ & $\begin{array}{c}- \\
18\end{array}$ & $\begin{array}{c}- \\
100\end{array}$ & $\overline{7}$ & $\begin{array}{c}- \\
100\end{array}$ & 23.2 & $\mathrm{p}<.001$ \\
\hline $\begin{array}{l}\text { Syncytial } \\
\text { knots }\end{array}$ & $\begin{array}{c}>30 \\
30-50 \\
>50\end{array}$ & $\begin{array}{c}12 \\
13 \\
- \\
\end{array}$ & $\begin{array}{c}48 \\
52 \\
- \\
\end{array}$ & $\begin{array}{c}- \\
11 \\
7 \\
\end{array}$ & $\begin{array}{c}- \\
61 \\
39 \\
\end{array}$ & $\begin{array}{l}- \\
2 \\
5 \\
\end{array}$ & $\begin{array}{c}- \\
29 \\
71 \\
\end{array}$ & 13.27 & $\mathrm{p}<.01$ \\
\hline $\begin{array}{l}\text { Stromal } \\
\text { fibrosis }\end{array}$ & $\begin{array}{l}<6 \\
>6\end{array}$ & $\begin{array}{c}25 \\
-\end{array}$ & $\begin{array}{c}100 \\
-\end{array}$ & $\begin{array}{c}11 \\
7\end{array}$ & $\begin{array}{l}61 \\
39\end{array}$ & $\begin{array}{l}2 \\
5\end{array}$ & $\begin{array}{l}29 \\
71\end{array}$ & 13.27 & $\mathrm{p}<.01$ \\
\hline
\end{tabular}

\begin{tabular}{|c|c|c|c|c|c|c|c|}
\hline Group & N & \multicolumn{2}{|c|}{ Term } & Pre-Term & X Value & P Value \\
\hline & & No. & \% & No. & & \multirow{2}{*}{ P $<.01$} \\
\hline Control & 50 & 39 & 78 & 11 & 22 & \multirow{2}{*}{7.43} & \multirow{2}{*}{} \\
Study & 50 & 26 & 52 & 24 & 48 & \\
\hline \multicolumn{7}{|c|}{} \\
\hline
\end{tabular}

Foetal outcome in relation to severity of PIH and placental pathology. The incidence of preterm delivery in the study group was more than 2 times higher than that in the control group. Within the study group $100 \%$ of eclampsia cases had preterm babies compared to $67 \%$ in the severe group, which was statistically highly significant. Foetal outcome in relation to the extent of infarction was as seen in table. Adverse foetal outcome was rare when infarction involved less than $10 \%$ of the placenta. Infarction of $20 \%$ or more was significantly associated with increased incidence of perinatal loss. The incidence of IUGR and perinatal loss was significantly higher with cytotrophoblastic proliferation (40\%), basement membrane thickening and paucity of vasculosyncytial membrane. Intrauterine death was significantly associated with increased incidence of syncytial knots and stromal fibrosis. Thus, the observations in the present study indicate that the placental histopathology is directly related to the severity of PIH and the adverse foetal outcome is well correlated with the extent of infarction and the villous lesions in the placenta.

\section{DISCUSSION}

In the present study, the incidence of pregnancy-induced hypertension was more in primi of the age group 20 to 29 years. This observation is in accordance with any other study on PIH, placental pathology and weight of placenta. In this study, the mean placental weight in the severe and eclampsia group was much less compared to the control group. The ratio of foetal weight to placental weight is called foetoplacental ratio. ${ }^{9}$ In this study, the $\mathrm{f} / \mathrm{p}$ ratio in the $\mathrm{PIH}$ group was almost equal to that in the control group. According to Fox, the considerable reserve capacity and potential for further growth makes it unlikely that the placental mass limits foetal weight, the placenta in fact usually being small because the baby is small. ${ }^{10}$ 


\section{Infarction}

The percentage of Infarction increased proportionately with the severity of hypertension. The true significance of excessive placental infarction is that it is the visible hallmark of a severely compromised uteroplacental vasculature. ${ }^{8}$ Retroplacental clot, the increased incidence of retroplacental clot in PIH placentas found in this study was previously observed by Fox. Calcification and intervillous thrombus observed to an equal extent in both the control and study groups re-emphasise the conclusion of Fox and Wentworth that this abnormality is of no pathological or clinical importance. 10 Microscopic villous lesions in pregnancyinduced hypertension, the cytotrophoblastic hyperplasia is due to syncytial damage subsequent to placental ischaemia. ${ }^{11}$ Thus, the intensity of the proliferative activity serves as a rough guide to the severity and duration of the ischaemia to which the trophoblast has been subjected. In the present study, the severe PIH and eclampsia group showed marked proliferation correlating well with the adverse foetal outcome in these groups.

An undue thickening of the basement membrane has been noted in placenta from cases of PIH, both on light microscopy and electron microscopy. The present study showed the placentas from severe and eclampsia group to have high basement membrane count as supported by Fox and Sodhi et al.12 A vasculosyncytial membrane consists of attenuated, anuclear syncytiotrophoblast stretched over and in close apposition to a sinusoidally dilated vessel. ${ }^{12}$ An unduly low number of vasculosyncytial membrane is a reflection of either villous immaturity or villous regressive changes. The low count observed in the present study must be a manifestation of villous regression. The strong correlation seen between the paucity of the membrane and the foetal adversities can be explained by the fact that this area of the villi is specially adapted for maternal foetal transfer. ${ }^{13}$ Fibrinoid necrosis of the placental villi evolves as a small blob of homogenous, strongly PAS positive material in the trophoblast gradually enlarging and invading the entire villi. The high fibrinoid necrosis accounted (73\%) in PIH placentas found in this study is supported by the observation of Fox, who attributed it to be an immunological reaction to circulating antitrophoblastic antibody. Syncytial knots are focal clumps of syncytial nuclei that protrude into the intervillous space from the surface of the villi. The formation of syncytial knots is greatly increased in villi, in which the foetal blood flow is reduced due to thrombosis or obliterative changes in the foetal arteries as a cause in intrauterine death in PIH. ${ }^{14,15}$ In the present study syncytial knots count was high in placentas with IUD, where the foetal stem artery showed obliteration. The importance of villous fibrosis is that it is a morphological hallmark of a reduced foetal villous perfusion. ${ }^{16}$ In the present study, only 12 out of 50 showed significant percentage of stromal fibrosis, of which 11 were found in association with IUD. The foetal stem artery showed obliterative changes in 5 of these placenta, which is supported by the studies of Fox.17

There was a decreasing gradient for foetal weight among patients with mild PIH, severe PIH and eclampsia, correlating well to the extent of placental pathology. ${ }^{18}$

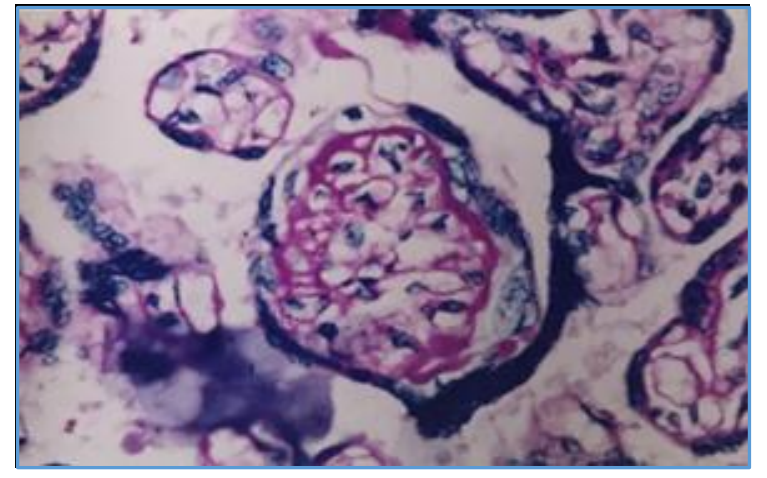

Figure 1. Prominent Cytotrophoblastic Membrane

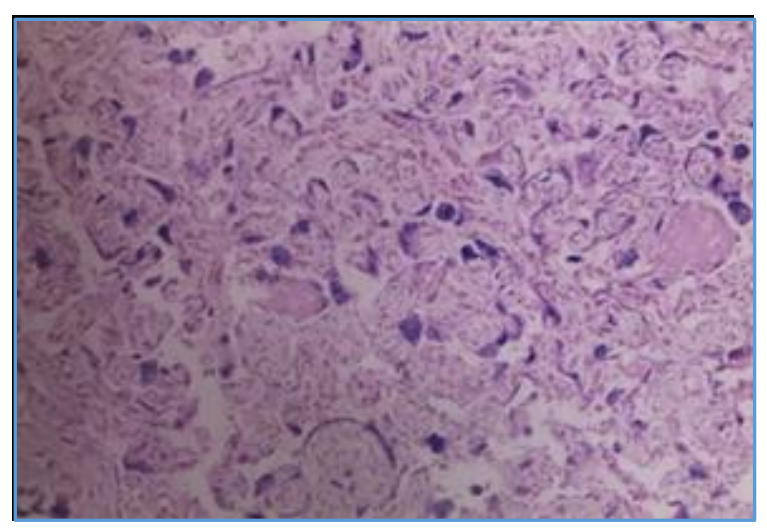

Figure 2. Infarction

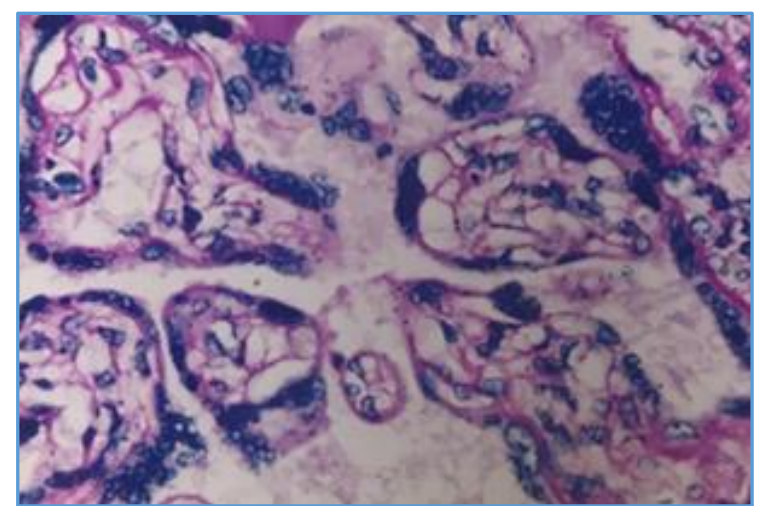

Figure 3. Syncytial Knots

\section{CONCLUSION}

The placental histopathological findings observed in this study suggests the existence of a reduced uteroplacental blood flow in pregnancy-induced hypertension. The abnormal placentation in PIH has been well established by many workers by their studies on the placental bed vasculature. As a response to the uteroplacental ischaemia there is vasoconstriction of the foetal stem arteries in the placenta, manifesting histologically as sclerosis of vessels and increased number of syncytial knots. The intrauterine growth retardation and foetal loss observed in PIH are also attributable to the defective uteroplacental perfusion. The fact that the placental bed vessels, which harbour the important pathological processes are not usually available for study is particularly distressing. However, placental histopathology with more emphasis on the technique of placental bed biopsy could compliment the clinical diagnosis or support the clinical suspicion in cases in which the blood pressure values do not meet the criteria for a diagnosis of 
preeclampsia. An accurate diagnosis is particularly desirable for research purposes and for counselling the patient on recurrence risk in future pregnancies.

\section{REFERENCES}

[1] Page EW. On the pathogenesis of pre-eclampsia and eclampsia. Journal of Obstetrics and Gynaecology of the British Commonwealth 1972;79(10):883-94.

[2] Benirschke K. Examination of the placenta. Obstetrics and Gynaecology 1961;18(3):309-33.

[3] Benirshke K. The placenta: How to examine it and what you can learn? Contemp Obstetpo Gynaecol 1981;17:117-9.

[4] Ghidini A, Salafia CM, Pezzullo JC. Placental vascular lesions and likelihood of diagnosis of preeclampsia. Obstetrics and Gynaecology 1997;90(4 Pt 1):542-5.

[5] Bewley S, Cooper D, Campbell S. Doppler investigation of utero-placental blood flow resistance in the second trimester: a screening study for pre-eclampsia and intra-uterine growth retardation. Br J Obst Gynaecol 1991;98(9):871-9.

[6] Khong TY, De Wolf F, Robertson WB. Inadequate maternal vascular response to placentation in pregnancy complicated by pre-eclampsia and by small-for-gestational age infants. British Journal of Obstetrics and Gynaecology 1986;93(10):1049-59.

[7] Kher AV, Zawar MP. Study of placental pathology in toxemia of pregnancy and its fetal implications. Indian Journal of Pathology and Microbiology 1981;24(4):245-51.

[8] Wallenburg HC, Stolte LA, Janssens J. Pathogenesis of placental infarction. I. A morphologic study in the human placenta. American Journal of Obstetrics and Gynaecology 1973;116(6):835-40.
[9] Hitschold T, Ulrich S, Kalder M, et al. Blood flow profile in the uterine artery. Co-relation with placental morphology and clinico- obstetrical data within the scope of pre-eclampsia. Z Geburtshilfe Neonatol 1995;199(1):8 -12.

[10] Boyd PA, Scott A. Quantitative structural studies on human placentas associated with pre-eclampsia essential hypertension and intrauterine growth retardation. Br J Obstet Gynaecol 1985;92(7):714-21.

[11] Fox H. The villous cytotrophoblast as an index of placental ischemia. Journal of Obstetrics and Gynaecology of the British Commonwealth 1964;71:885-93.

[12] Fox H. The incidence and significance of vasculosyncitial membrane in the human Placenta. Journal of Obstetrics and Gynaecology of the British Commonwealth 1967a;74:28-34.

[13] Sodhi S, Mohan H, Jaiswal TS, et al. Placental Pathology in pre-eclampsia eclampsia syndrome. Indian Journal of Pathology and microbiology 1990;33(1):11-6.

[14] Cibils LA. The placenta and newborn infant in hypertensive conditions. Am J Obstet Gynaecol 1974;118(2):256-70.

[15] Chakravorthy AP. Foetal and placental weight changes in normal pregnancy and pre-eclampsia. J Obstet Gynaecol Br Commonw 1967;74(2):247-53.

[16] Fox H. The morphological basis of placental insufficiency. J Obstet Gynaecol India 1975;25(4):44150 .

[17] Zacutti A, Borruto F, Bottacci G, et al. Umbilical blood flow and placental pathology. Clin Exp Obstet Gynaecol 1992;19(1):63-9.

[18] Bhatia A, Sharma SD, Jalnawalla SF, et al. A comparative study of placental and foetal outcome. Indian J Pathol Microbiol 1981;24(4):277-83. 